\title{
USING A NPWE MODEL OBSERVER TO ASSESS SUITABLE IMAGE QUALITY FOR A DIGITAL MAMMOGRAPHY QUALITY ASSURANCE PROGRAMME
}

\author{
P. Monnin ${ }^{1,2, *}$, F. O. Bochud ${ }^{1}$ and F. R. Verdun ${ }^{1}$ \\ ${ }^{1}$ Institute for Radiation Physics (IRA), CHUV - UNIL, Grand Pré 1, 1007 Lausanne, Switzerland \\ ${ }^{2}$ Haute Ecole Cantonale Vaudoise de la Santé (HECVSanté), Filière TRM, Avenue de Beaumont 21, \\ 1011 Lausanne, Switzerland \\ *Corresponding author: pascal.monnin@chuv.ch
}

\begin{abstract}
A method of objectively determining imaging performance for a mammography quality assurance programme for digital systems was developed. The method is based on the assessment of the visibility of a spherical microcalcification of $0.2 \mathrm{~mm}$ using a quasi-ideal observer model. It requires the assessment of the spatial resolution (modulation transfer function) and the noise power spectra of the systems. The contrast is measured using a 0.2-mm thick Al sheet and Polymethylmethacrylate (PMMA) blocks. The minimal image quality was defined as that giving a target contrast-to-noise ratio (CNR) of 5.4. Several evaluations of this objective method for evaluating image quality in mammography quality assurance programmes have been considered on computed radiography (CR) and digital radiography (DR) mammography systems. The measurement gives a threshold CNR necessary to reach the minimum standard image quality required with regards to the visibility of a $0.2-\mathrm{mm}$ microcalcification. This method may replace the CDMAM image evaluation and simplify the threshold contrast visibility test used in mammography quality.
\end{abstract}

\section{INTRODUCTION}

Implementation of an effective quality control protocol in mammography is of the greatest importance for obtaining the best image quality at the lowest possible dose to minimise the risk. Quality control is therefore essential to ensure the equipment used is reliable and consistent to maintain radiation doses as low as reasonably achievable while optimising image quality and performance in mammography. Quality assurance in mammography has received increasing attention as an essential element of a successful breast cancer screening programme. The European Commission developed and published the European Protocol for the Quality Control of the Technical Aspects of Mammography Screening (EPQCM) and the European Guidelines for Quality Assurance in Mammography Screening in $1993^{(1,2)}$. The American College of Radiology developed and published Mammography Quality Control Manuals in $1990^{(3)}$ and the Breast Imaging Reporting and Data System (BIRADS) in $1993^{(4)}$. One approach to control the ability of a mammography system to reach a minimal image quality for a given dose is given in the fourth edition of the European Guidelines for quality assurance in breast cancer screening and diagnosis.

This study focuses on the "threshold contrast visibility' protocol (section 2.4.1 of the EPQCM), in which human observers score images of a CDMAM phantom. Because of the variable and subjective nature of the human-observer decision, several studies revealed an important image phantom and observer variability in the visibility of the targets.
This variability may affect the result of the threshold contrast visibility test and limits the ability of accurately measuring system performance ${ }^{(5)}$. In particular, partial volume effects and the viewing environment can affect the efficiency of visual scoring of CDMAM phantom images. Software tools have been developed to automatically evaluate CDMAM images and increase scoring efficiency. Although they give a good correlation with human observer performance, they also demonstrate differences in detection sensitivity ${ }^{(6)}$.

Digital mammography systems with inherent digital data provide the possibility of using quantitative evaluations of image quality, which do not rely on a human-observer decision threshold. The standardised measurements of mammographic modulation transfer function (MTF) and noise power spectra (NPS) ${ }^{(7)}$ provide the basis for a more objective and quantitative evaluation of image performance. In this study, a nonprewhitening model observer weighed for the response of the eye (NPWE) was applied to assess the image quality and verify the adequacy of automatic exposure control (AEC) dose level settings in the mammography quality control programme. The use of model observers demonstrated its ability to provide good reproducibility in the indication of clinical performance in digital mammography ${ }^{(7,8)}$. Like the 'object thickness and tube voltage compensation' test of the EUREF protocol, the study control method is based on the contrast-to-noise (CNR) measurement with an aluminium object of $0.2-\mathrm{mm}$ thickness on top of 


\section{P. MONNIN ET AL}

PMMA plates of 20, 40 and $60 \mathrm{~mm}$. The result indicates whether the current AEC on the system provides a sufficient dose for a threshold image quality. This method may replace the CDMAM image evaluation and simplify the threshold contrast visibility test used in mammography quality controls.

\section{METHOD}

Four digital mammography systems were included in the study: two CR Kodak CR 975 EHR-M and EHR-M2, a flat-panel GE Senographe Essential and a scanned photon counter Sectra MDM.

CNR images were obtained at the clinical beam quality obtained automatically with the AEC. Theoretical CNR was calculated as defined for a NPWE model observer assuming spatial stationarity in order to be expressed in the Fourier domain. It corresponds to the detectability index $\left(d^{\prime}\right)$ of the NPWE model for a signal known case with Gaussian, stationary noise and background ${ }^{(9)}$ :

$\mathrm{CNR}=\frac{\sqrt{2 \pi} C \int_{0}^{\infty} S^{2}(f) \operatorname{MTF}^{2}(f) \operatorname{VTF}^{2}(f) f \mathrm{~d} f}{\sqrt{\int_{0}^{\infty} S^{2}(f) \mathrm{MTF}^{2}(f) \operatorname{VTF}^{4}(f) \operatorname{NNPS}(f) f \mathrm{~d} f}}$

where $C$ is the normalised contrast between the aluminium foil and the PMMA background, $S(f)$ is the object transfer function, $\operatorname{MTF}(f)$ is the modulation transfer function, $\operatorname{NNPS}(f)$ is the normalised noise power spectrum and $\operatorname{VTF}(f)$ is the visual transfer function of the human eye.

The object transfer function for a spherical calcification with radius $R$ is given by:

$$
S(f)=4 \pi R^{3}\left(\frac{\sin (2 \pi f R)}{(2 \pi f R)^{3}}-\frac{\cos (2 \pi f R)}{(2 \pi f R)^{2}}\right)
$$

To account for the visual characteristics of the observer, a VTF which can account for some degradation of the information perceived by the observer was introduced ${ }^{(10)}$ :

$$
\operatorname{VTF}(f)=f^{1.8} \exp \left(-0.6 f^{2}\right)
$$

The normalised contrast between a $0.2-\mathrm{mm}$ aluminium foil and PMMA blocks of 20,40 and $60 \mathrm{~mm}$ was obtained using the following formula:

$$
\begin{aligned}
C=\frac{1}{0.2} \cdot \frac{\text { mean pixel value (PMMA) }}{(\text { mean pixel value }(\mathrm{Alu})} \\
+ \text { mean pixel value }(\mathrm{Alu})) / 2
\end{aligned}
$$

The MTF of the different systems (Figure 1) was determined as specified in IEC $62220-1-2^{(11)}$. The impulse response was obtained from the image of a $500-\mu \mathrm{m}$ thick sharp edged tungsten plate tilted at about $2^{\circ}$ with respect to the lines or columns of the pixels, and positioned along the central axis of the $\mathrm{X}$-ray beam, close to the surface of the detectors.

The NPS for the systems (Figure 2) was assessed according to the international standard IEC 62220$1-2$. For the calculation, square areas of $512 \times 512$ pixels were selected at the centre of the linearised images obtained without the aluminium foil.

System response curves were determined with uniform images acquired at different exposure levels using the anode-target combination used on the different systems for a given PMMA thickness to plot the relationship between the mean pixel value and the tube load (in mAs) incident on the detectors. These curves were used to express the image pixel values into X-ray air kerma levels for the MTF and NPS calculations.

The threshold CNR was chosen at 5.4. It corresponds to a probability of correct observer decision higher than $96.8 \%{ }^{(12)}$.

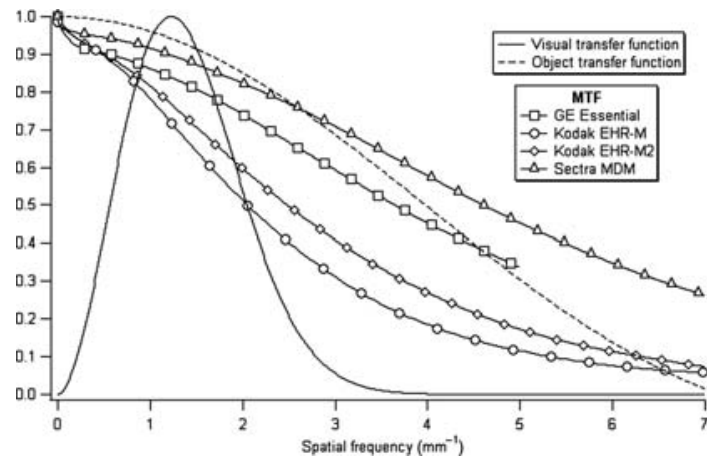

Figure 1. MTF of the four mammography systems and visual and object transfer functions.

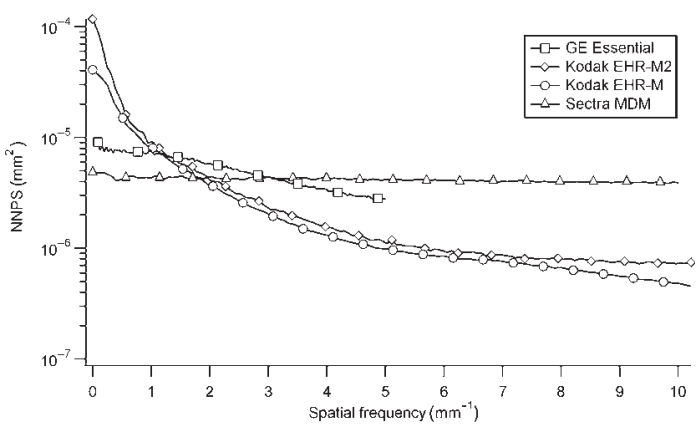

Figure 2. NNPS of the four mammography systems for a detector dose of $50 \mu \mathrm{Gy}$. 


\section{RESULTS AND DISCUSSION}

Figures 3 and 4 show the CNR measured on two mammography systems (GE Senographe Essential and Kodak CR 975 EHR-M) as a function of mean glandular breast dose for three PMMA thicknesses (20, 40 and $60 \mathrm{~mm})$. The CNR obtained at the AEC settings are reported. The first system is an example of a mammography system, which can easily reach the threshold CNR without overcoming the mean glandular dose (MGD) limit for any thickness. The second system cannot reach the threshold CNR at 40 and $60 \mathrm{~mm}$ without overcoming the MGD limits specified in the EUREF $(2 \mathrm{mGy}$ at $40 \mathrm{~mm}$ and $4.5 \mathrm{mGy}$ at $60 \mathrm{~mm}$ ).

Figure 5 shows the theoretical CNR curves of the four mammography systems calculated from equation (1) as a function of a microcalcification diameter. These curves were obtained for the AEC settings used on these systems for a PMMA thickness of $40 \mathrm{~mm}$. The graph shows the threshold CNR of 5.4 for the visibility of a $0.2 \mathrm{~mm}$ aluminium

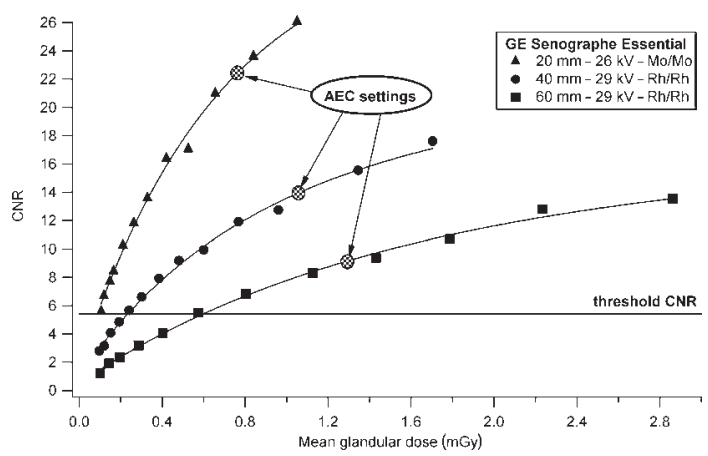

Figure 3. CNR measured on the GE Senographe Essential system as a function of mean glandular dose for three PMMA thicknesses (20, 40 and $60 \mathrm{~mm})$.

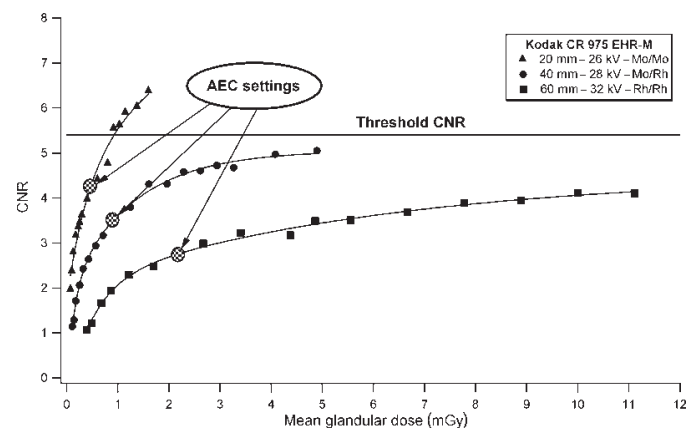

Figure 4. CNR measured on the Kodak CR 975 EHR-M system as a function of mean glandular dose for three PMMA thicknesses $(20,40$ and $60 \mathrm{~mm})$ sphere. The system curves depend on image dose and therefore on the AEC setting. Only the systems with a curve reaching the quadrant in the upper left corner can provide the minimum standard image quality required with regards to the visibility of a $0.2-\mathrm{mm}$ microcalcification.

The results of this study concern the use of a NPWE model observer to assess the image quality and AEC dose level in the mammography quality control programme. This control procedure was successfully applied to all types of digital mammography systems. It is a different approach than setting a threshold image quality, is easier to implement than the EUREF protocol and is less time-consuming. The threshold contrast visibility determined with the CDMAM phantom needs indeed six images for every condition and three experienced observers who have to analyse at least five diameters in detail. The use of a NPWE model observer needs only three images per thickness and can easily be calculated by a software (no additional human resources are needed).

A general conclusion for imaging systems is that the observer CNR is linearly proportional to object contrast and size ${ }^{(13)}$. This behaviour is in agreement with both NPWE model observer CNR and the European protocol. Some bias due to linear signal trends such as heel or geometric effects can be introduced in the value of the observer CNR. An appropriate removal of these biases in the data was necessary for CR system images which are not corrected by a flat field procedure. These biases on noise measurement were limited by using small signal areas for noise measurement and with images obtained by exposing the CR cassette using half load under normal condition and half load with cassette rotated $180^{\circ}$ in the bucky. Therefore, the precision and reproducibility of this quantitative imaging performance evaluation were much higher than scoring by human observers. Large acceptability of this CNR observer evaluation technique will probably need a further validation with a systematic comparison using the test from the EUREF protocol.

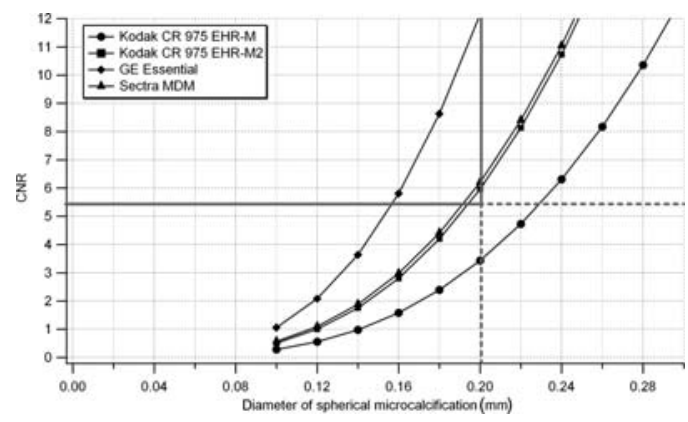

Figure 5. Theoretical CNR of four digital mammography systems as a function of a microcalcification diameter. 


\section{P. MONNIN ET AL}

\section{CONCLUSION}

The use of a NPWE model observer to assess the image quality and AEC dose level in a mammography quality control programme demonstrated its ability to provide good reproducibility in the indication of clinical performance. This model for assessing the imaging performance for digital mammography systems indicated by an easy test the desirable detector dose that has to be used for the required visibility of a $0.2 \mathrm{~mm}$ microcalcification. This method was successfully implemented to replace the CDMAM image evaluation and simplify the threshold contrast visibility test used in mammography quality controls.

\section{REFERENCES}

1. The European protocol for the quality control of the technical aspects of mammography screening. CEC Report EUR 14821 (1993).

2. de Wolf, C. J. M. and Perry, N. M. (eds). European Guidelines for Quality Assurance in Mammography Screening, second edn. (Luxembourg: European Commission, Europe against Cancer Programme) (1996).

3. Hendrick, R. E. et al. Mammography Quality Control: Radiologist's Manual, Radiologic Technologist's Manual, Medical Physicist's Manual. (Reston., VA: American College of Radiology) (1990).

4. Kopans, D. B. et al. Breast Imaging Reporting and Data System (BIRADS), first edn (Reston, VA: American College of Radiology) (1993).

5. Van Metter, R., Heath, M. and Fletcher-Heath, L. Applying the European protocol for the quality control of the physical and technical aspects of mammography screening threshold contrast visibility assessment to digital systems. Proc. SPIE 6142, 18-38 (2006).

6. Fletcher-Heath, L. and Van Metter, R. Quantifying the performance of human and software CDMAM phantom image observers for the qualification of digital mammography systems. Proc. SPIE 5745, 486-498 (2005).

7. Marshall, N. W. A comparison between objective and subjective image quality measurements for a full field digital mammography system. Phys. Med. Biol. 51, 2441-2463 (2006).

8. Segui, J. A. and Zhao, W. Amorphous selenium flat panel detectors for digital mammography: validation of a NPWE model observer with CDMAM observer performance experiments. Med. Phys. 33, 3711-3721 (2006).

9. Wagner, R. F., Brown, D. G. and Pastel, M. S. Application of information theory to the assessment of computed tomography. Med. Phys. 6, 83-94 (1979).

10. Burgess, A. E. Statistically defined backgrounds: performance of a modified nonprewhitening observer model. J. Opt. Soc. Am. A. 11, 1237-1242 (1994).

11. International Electrotechnical Commission. IEC 62220-1-2 2005 Medical Electrical Equipment Characteristics of Digital X-ray Imaging Devices - Part 1-2: Determination of the Detective Quantum Efficiency - Mammography Detectors (Geneva, Switzerland: International Electrotechnical Commission).

12. Desponds, L., Depeursinge, C., Grecescu, M., Hessler, C., Samiri, A. and Valley, J.-F. Image quality index (IQI) for screen-film mammography. Phys. Med. Biol. 36, 19-33 (1991).

13. Thomas, J. A., Chakrabarti, K., Kaczmarek, R. and Romanyukha, A. Contrast-detail phantom scoring methodology. Med. Phys. 32, 807-814 (2005). 